\title{
FAST AXONAL TRANSPORT IN PERMEABILIZED LOBSTER GIANT AXONS IS INHIBITED BY VANADATE ${ }^{1}$
}

\author{
DAVID S. FORMAN, ${ }^{*}, 2$ KURT J. BROWN,,* AND DAVID R. LIVENGOOD $\ddagger$ \\ * Department of Anatomy, Uniformed Services University of the Health Sciences and $\ddagger$ Armed Forces Radiobiology Research \\ Institute, Bethesda, Maryland 20814
}

Received October 22, 1982; Revised January 17, 1983; Accepted January 18, 1983

\begin{abstract}
We have developed a method for permeabilizing axons and reactivating the fast transport of microscopically visible organelles. Saltatory movements of organelles in motor axons isolated from lobster walking legs were observed using Nomarski optics and time-lapse video microscopy. In the center of the axon most of the particles and mitochondria moved in the retrograde direction, but immediately below the axolemma the majority moved in the anterograde direction. When axons were permeabilized with $0.02 \%$ saponin in an adenosine 5 '-triphosphate (ATP)-free "internal" medium, all organelle movement ceased. Saltatory movements resembling those in intact axons immediately reappeared upon the addition of MgATP. Very slight movement could be detected with ATP concentrations as low as $10 \mu \mathrm{M}$, and movement appeared to be maximal with 1 to $5 \mathrm{mM}$ ATP. Vanadate, which does not affect axonal transport in intact axons, inhibited the reactivated organelle movements in permeabilized axons. Movement was rapidly and reversibly inhibited by 50 to $100 \mu \mathrm{m}$ sodium orthovanadate. The effects of vanadate, including the time course of inhibition, its reversibility, and its concentration dependence, are consistent with the hypothesis that a dyneinlike molecule may play a role in the mechanism of fast axonal transport.
\end{abstract}

Fast axonal transport can be directly visualized in living axons by light microscopy (Smith, 1971, 1977, 1980; Kirkpatrick et al., 1972; Cooper and Smith, 1974; Forman et al., 1977a, b; Forman and Shain, 1981; Allen et al., 1982a, b; Forman, 1982c; reviewed in Grafstein and Forman, 1980). Microscopically visible organelles move in both the anterograde and retrograde directions by saltatory movements similar to those seen in a variety of other cells (Rebhun, 1972; Forman, 1982b). The cellular mechanism of fast transport is poorly understood. Many experimental probes that might be useful for studying

\footnotetext{
${ }^{1}$ This work was supported by the Uniformed Services University of the Health Sciences, Protocols RO7035 and RO7032, and United States Public Health Service Grant GM30450. The opinions or assertions contained herein are the private ones of the authors and are not to be construed as official or reflecting the views of the Department of Defense or the Uniformed Services University of the Health Sciences. We greatly appreciate a loan of equipment from the Naval Medical Research Institute, Bethesda, Maryland. We wish to thank Mark Promersberger for excellent technical assistance, Dr. Mark Adelman for valuable discussion, and Clayton Cisar and Ed Lundgren, Laboratory Animal Medicine Department, Uniformed Services University of the Health Sciences, for help in procuring lobsters.

${ }^{2}$ To whom correspondence should be addressed at Department of Anatomy, Uniformed Services University of the Health Sciences, School of Medicine, 4301 Jones Bridge Road, Bethesda, MD 20814.
}

the mechanism of transport do not cross the axonal plasma membrane. One approach to circumvent this problem that has been useful for studying other types of cell motility is to destroy the permeability barrier of the plasma membrane with detergents in a medium that permits reactivation of the movement. Detergent-permeabilized cells have proved useful for analyzing the mechanisms underlying other types of cell motility, such as muscle contraction, ciliary motion, chromosome movement, and cytokinesis (Cande and Wolniak, 1978; Cande et al., 1981). Recently, methods have been reported for reactivating saltatory movement in fibroblasts (Forman, 1981; 1982b), chromatophores (Clark and Rosenbaum, 1982; Stearns and Ochs, 1982) and neuroblastoma cells (Stearns and Buggs, 1981) permeabilized with detergent, and in isolated squid axoplasm (Brady et al., 1982). In this paper we describe a method for permeabilizing lobster axons with saponin and reactivating fast transport with exogenous ATP. We have used this method to study the effects of vanadate, an inhibitor of dynein (the ATPase involved in the movement of cilia and flagella, reviewed in Warner and Mitchell, 1980). Vanadate, which cannot normally enter living axons, reversibly inhibits fast organelle transport in the permeabilized axons. This finding suggests that a cytoplasmic dynein-like molecule may play a role in the mechanism of fast axonal transport. 


\section{Materials and Methods}

Isolation of giant axons. Lobsters (Homarus americanus) from commercial sources, weighing 0.5 to $1 \mathrm{~kg}$, were maintained in aerated holding tanks in artificial sea water at $4^{\circ} \mathrm{C}$ for up to 3 days. Walking legs were removed immediately before dissection and placed in an "external" medium containing $450 \mathrm{~mm} \mathrm{NaCl}, 15 \mathrm{~mm} \mathrm{KCl}$, $25 \mathrm{mM} \mathrm{CaCl}_{2}, 4 \mathrm{mM} \mathrm{MgSO}_{4}, 4 \mathrm{mM} \mathrm{MgCl}_{2}$, and $10 \mathrm{mM}$ HEPES (sodium salt), $\mathrm{pH}$ 7.4. Motor nerves in the meropodite (Wiersma, 1961) were exposed, and the proximal and distal ends of 3- to 5-cm-long segments were tied with surgical threads of different diameters so that the direction of movement could later be identified. The nerves were placed in 85- $\mathrm{mm}$ plastic Petri dishes containing about $15 \mathrm{ml}$ of external medium and were anchored by pushing the suture ends into a 5-mm-thick layer of Sylgard (Dow Corning) on the bottom. Single giant motor axons or adherent pairs were isolated using a Zeiss microsurgical dissecting microscope and darkfield illumination. In most experiments the axons studied were either the "fast closer" or one of the two "opener" axons to the dactylopodite (Wiersma, 1961; Kravitz et al., 1965); there was no obvious difference in movement between the different types of axons. The diameters of the axons ranged from 30 to $50 \mu \mathrm{m}$.

A coverslip longer than the nerve segment was slipped underneath it, and the medium was gently removed with a syringe. The coverslip with the adhering axon was then quickly inverted and lowered onto a pool of medium on a spacer slide. The spacer slide consisted of a $74 \mathrm{~mm} \times$ $50 \mathrm{~mm}$ glass slide to which two $60-\mathrm{mm}$ long no. 1 coverslips were glued with Permabond 910 adhesive, leaving a gap of $10 \mathrm{~mm}$ between them. The sides of the coverslip were sealed with melted Kronig wax. Solutions were changed by pipetting fluid at one end of the coverslip and absorbing it at the other end with filter paper.

Video microscopy. The axons were observed with Nomarski differential interference contrast optics using a Zeiss basic microscope with a $\times 40$ planachromatic objective (numerical aperture $=0.65$ ). A Calflex heat reflection filter was used to protect the specimen, and a Zeiss VG9 filter provided monochromatic (546 $\pm 5 \mathrm{~nm}$ ) light. Best results were obtained with the polarizer parallel to the axon. The microscopic image was magnified and recorded using a video microscopy system (Willingham and Pastan, 1978) consisting of a highly sensitive SIT video camera (RCA TC1030H), a time-lapse videotape recorder (Panasonic NV-8030 or NV-8050), and a monitor (RCA TC1209). Time was continuously displayed on the video record by a date and time generator (RCA TC1440B). The axons were observed at a magnification of $\times 4500$ on the monitor screen and recorded in time-lapse mode so that time was compressed by a factor of 9 . Temperature, which was monitored by a thermistor taped to the stage, ranged from $21^{\circ}$ to $24^{\circ} \mathrm{C}$.

To measure the proportion of organelles moving in the retrograde and anterograde directions, the numbers of particles crossing a vertical line at the center of the monitor screen were counted for 5-min segments of videotape record. Elongated organelles, presumed to be mitochondria, were counted separately. To measure the frequency of organelle movements, the monitor screen was covered by a $45 \times 45 \mathrm{~mm}$ mask (equivalent to an area of $100 \mathrm{~mm}^{2}$ ). Long saltations and "tugging movements" (see "Results") with net displacements of greater than $1 \mu \mathrm{m}$ were counted during successive 1 -min periods of videotape record. Counts from four equally spaced regions (totaling $40 \%$ of the screen area) were added together to give a representative index of the frequency of organelle movements (see Forman, 1982a). Particle velocities were measured from videotape records using a $610 E$ Video Pointer (Colorado Video, Boulder, Colorado).

Permeabilization. Organelle movements inside an axon were observed in external medium to provide a base line. The axon was then incubated for $10 \mathrm{~min}$ in an "internal" medium (modified from Abercrombie et al., 1981) that contained $300 \mathrm{~mm}$ potassium aspartate, $20 \mathrm{~mm}$ sodium aspartate, $344 \mathrm{~mm}$ glycine, $10 \mathrm{mM} \mathrm{MgCl} 2,5 \mathrm{mM}$ EGTA, and $10 \mathrm{~mm}$ HEPES, pH 7.3. The axon was then permeabilized by incubation for $15 \mathrm{~min}$ in internal medium plus $0.02 \%$ saponin (Sigma) and $1 \%$ dimethyl sulfoxide (DMSO). The saponin was added to the medium from a stock solution of $2 \%(\mathrm{w} / \mathrm{v})$ saponin dissolved in DMSO. DMSO alone (1\%) did not permeabilize the axon or noticeably affect transport. The axon was then perfused with various reactivation media consisting of additional components (described under "Results") dissolved in internal medium without saponin or DMSO.

\section{Results}

Fast axonal transport of microscopically visible organelles in intact axons. With conventional Nomarski optics, numerous organelles can be seen moving inside living lobster giant axons. The sheath surrounding each isolated axon, consisting of several layers of thin glial cells and collagen fibers, did not excessively interfere with good visualization of organelles in the axoplasm (Fig. 1). The organelles and their movements resembled those that have been described in other types of axons (Smith, 1971, 1972, 1977, 1980; Kirkpatrick et al., 1972; Cooper and Smith, 1974; Forman et al., 1977a, b; reviewed in Grafstein and Forman, 1980; Forman, 1982b). Briefly, two classes of organelles could be distinguished: elongated mitochondria and small, round or ellipsoidal, vesicular "particles" (Figs. 1 and 2, $a$ to $c$ ). ${ }^{3}$ Mitochondria varied in length from a few micrometers to longer than $40 \mu \mathrm{m}$ and were usually aligned parallel to the long axis of the axon. Mitochondria were found distributed throughout the axoplasm but were especially concentrated immediately below the axonal plasma membrane. More particles than mitochondria were seen moving (Table I). The movement of both particles and mitochondria appeared to be similar to the saltatory organelle

\footnotetext{
${ }^{3}$ By the use of the Allen video-enhanced contrast, differential interference contrast (AVEC-DIC) technique, Allen et al. (1982a, b) have reported detecting three classes of organelles: mitochondria, "medium" size particles, and "small" particles. They propose that the small particles, which move predominantly in the anterograde direction, represent 30 - to $50-\mathrm{nm}$ vesicles that have not previously been observed by light microscopy (Allen et al., 1982a, b; Brady et al., 1982; Fahim et al., 1982). In our study, like all previous studies using conventional microscopy, "small" vesicles were not detected; all particles described in this paper were of the "medium" type. The implications of this are considered further under "Discussion."
} 


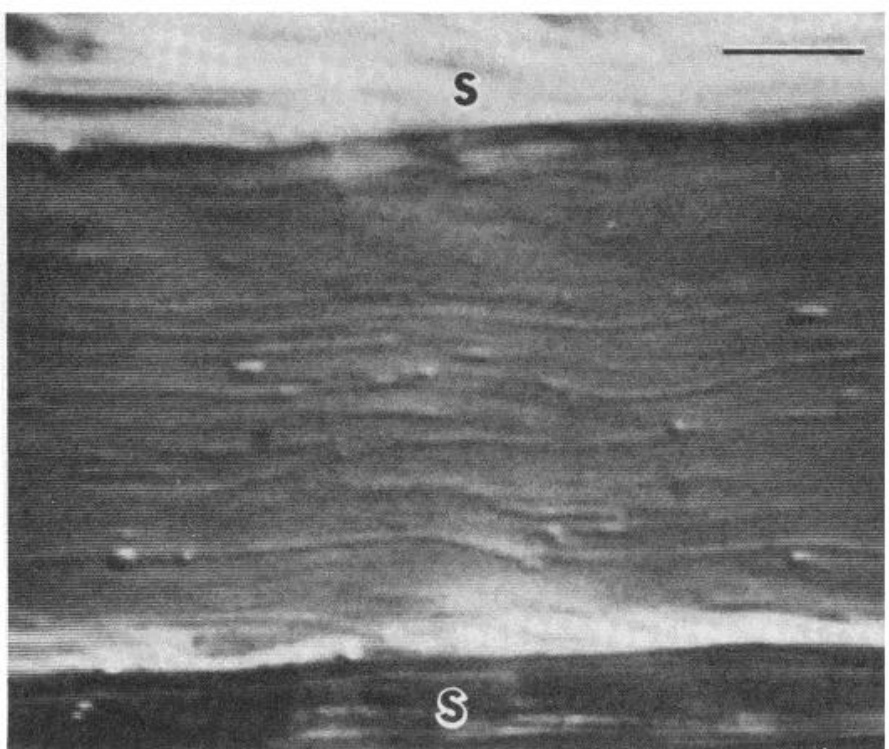

Figure 1. Low magnification view of isolated lobster giant axon $(b a r=10 \mu \mathrm{m})$. A sheath (s) surrounds the axon. The axon contains elongated mitochondria and small spherical or ellipsoidal vesicles ("particles").

movements seen in other types of cells (Rebhun, 1972). The organelles moved in straight translocations parallel to the long axis of the axon (Fig. 2, $a$ to $c$ ), with velocities usually between 0.3 and $2.4 \mu \mathrm{m} / \mathrm{sec}$ (Table I). The movement was often interrupted by pauses of variable duration or by brief reversals of direction. Each organelle moved predominantly in one "major" direction (Forman et al., 1977a).

Despite overall similarities, there were some interesting differences between the patterns of organelle movement that we observed in lobster axons and those that have been described in other types of axons: (1) Although many mitochondria in the lobster axon were stationary, mitochondrial movement in these axons appeared to be more frequent (Table I) than in vertebrate axons (Cooper and Smith, 1974; Forman et al., 1977a). (2) In both vertebrate (Cooper and Smith, 1974; Forman et al., 1977a, b; Smith 1971, 1972, 1980) and crustacean axons (Smith, 1977; Adams, 1982) the great majority of the particles that are large enough to be seen with conventional optics have been seen to move in the retrograde direction, and no regional differences in particle direction have been reported. However, we found a regional difference in the lobster axons: in the interior of the axon more than $80 \%$ of the particles moved toward the cell body, but immediately inside the plasma membrane a majority of the particles (almost 70\%) moved in the anterograde direction (Fig. 3; Table I). The width of the zone below the axolemma where anterograde movement predominates appeared to be less than $1 \mu \mathrm{m}$. The mean velocity of retrograde particle movement (Table I) was the same in the interior and at the periphery of the axon; this was also true of the mean anterograde velocities ( $t$ tests, $p>$ 0.05 ). The mean retrograde velocity was more than twice as fast as the anterograde velocity (Table I). Mitochondria also showed more retrograde movement in the center and more anterograde movement near the surface (Table
I). In both regions the proportion of mitochondria moving in each direction was similar to the proportion of particles moving in the same direction.

When the external medium was replaced by internal medium before saponin treatment, there was no change in the velocities, frequency, or direction of particle movement. Movement continued for more than $1 \mathrm{hr}$ in internal medium without any obvious changes in organelle movement. Intracellular recordings in other axons confirmed that the axons were reversibly depolarized in internal medium, as would be expected from the high $\mathrm{K}^{+}$concentration. These results are consistent with previous demonstrations that fast transport is not affected by membrane depolarization (see Grafstein and Forman, 1980).

ATP-dependent fast transport of organelles in axons permeabilized with saponin. The permeabilization procedure produced a reproducible series of effects on axonal structure and organelle movement. Between 5 and 10 min after the addition of the saponin, several changes occurred that indicated breakdown of the plasma membrane permeability barrier. Some mitochondria developed a series of constrictions and periodic swellings resembling a string of beads (Fig. 2d), whereas others fragmented. Large empty vacuoles were rarely seen in normal axoplasm, but when such vacuoles were present they lost refractility and lysed during the permeabilization. Small particles did not show any obvious lysis or morphological change, but their saltatory movements decreased markedly. By $15 \mathrm{~min}$ after the arrival of the saponin, virtually all saltatory movement had ceased. Organelles remained immobile, without any Brownian movement. No additional changes were seen if the axon was left in the saponin-containing medium for up to an hour.

There was also no movement if the medium was replaced by saponin-free internal medium without ATP. However, when the axon was perfused with internal medium containing $1 \mathrm{~mm}$ MgATP, saltatory organelle movement dramatically reappeared (Figs. 2 and 4). The reactivated movements generally resembled those seen in intact axons. Although saltations tended to be shorter and slower than normal, some particles traversed the entire field of view $(40 \mu \mathrm{m})$. Movement occurred in both the anterograde and retrograde directions. Most of the moving organelles were particles, but mitochondrial saltations were also observed. The number of moving particles was variable but was always less than in the intact axon (Fig. 4). The number of movements during the first 5 min of reactivation with $1 \mathrm{~mm}$ ATP averaged $33 \%$ ( $\mathrm{SEM}=4 \% ; N=6$ ) of the number seen during the $5 \mathrm{~min}$ before the addition of saponin. The frequency of the movements declined with time, but usually movement could still be seen more than an hour after the first addition of ATP. However, if the medium was replaced by internal medium without ATP, all movement stopped within $2 \mathrm{~min}$. Movement could then be reactivated again by perfusion with medium containing ATP. Movement was not reactivated by 1 to $5 \mathrm{~mm}$ concentrations of adenosine monophosphate or by the nonhydrolyzable ATP analogue 5-adenylimidodiphosphate (AMP-PNP). A small number of distinct ATP-dependent movements could be detected with ATP concentrations as low as 10 

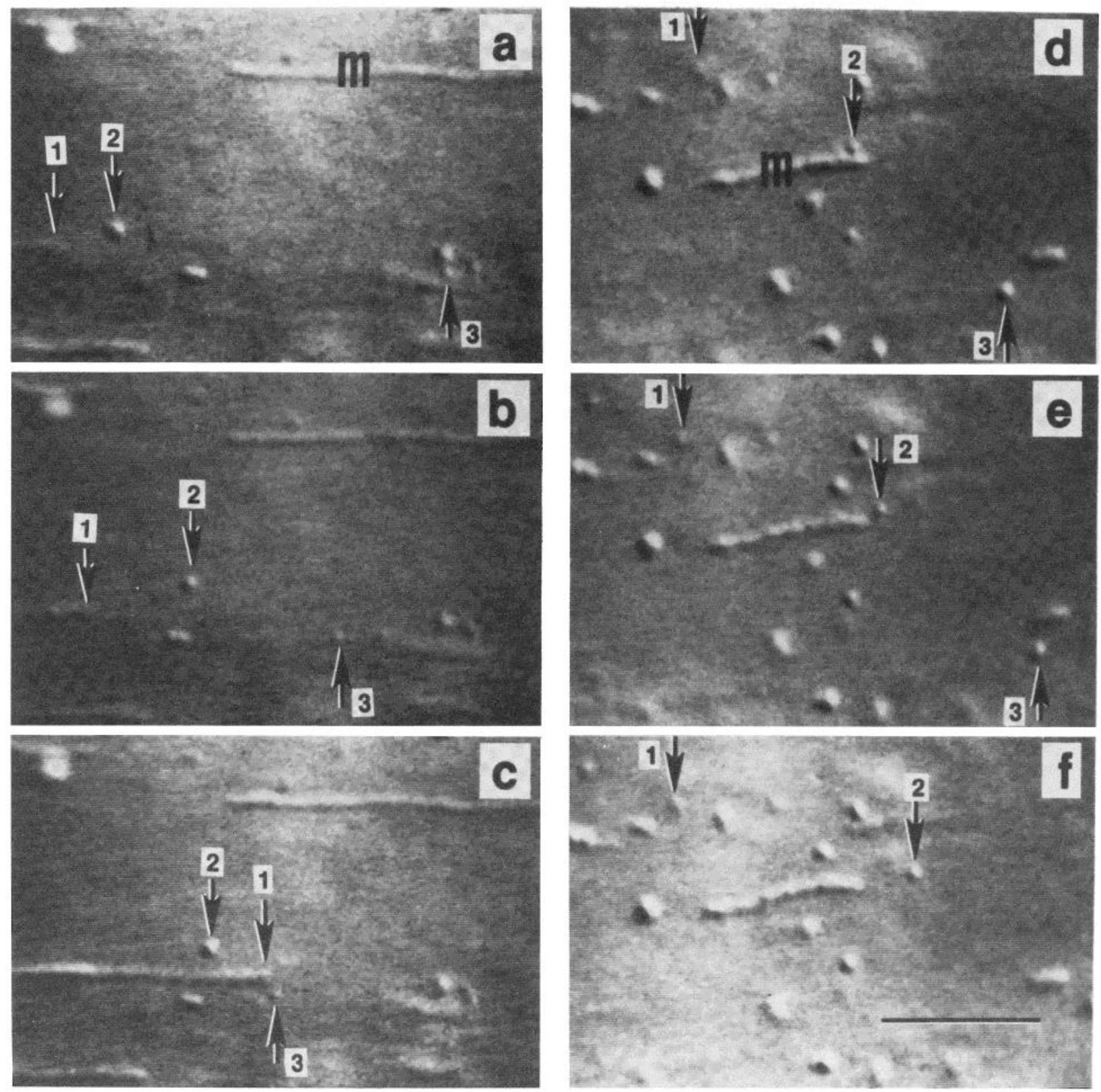

Figure 2. Saltatory movement in a lobster motor axon before and after permeabilization. Panels $a$ to $c$ demonstrate saltatory movement in the interior of a living isolated axon; $d$ to $f$ show the same axon after permeabilization with saponin and demonstrate organelle movement reactivated with ATP. The magnification of each panel is the same; the calibration bar in $f=10 \mu$ m. Retrograde movement is to the left. In $a$ to $c$ which were taken at 10-sec intervals, one particle (3) moved in the retrograde direction, while a particle (2) and a mitochondrion (1) moved in the anterograde direction. Another mitochondrion ( $m$ in $a$ ) remained stationary. $d$ to $f$ show the same axon after permeabilization with saponin as described under "Materials and Methods." Saponin-free medium containing $0.2 \mathrm{~mm}$ MgATP was added $1 \mathrm{~min}$ before $d$. $d$ to $f$ show successive 10 -sec intervals. Two particles ( 2 and 3 ) show saltations in the anterograde direction. Between $e$ and $f$ particle 3 moved out of the field of view. The smaller excursion of particle 1 reflects a "tugging" movement (see "Results"). The beaded appearance of the stationary mitochondrion $(m$ in $d)$ is typical of permeabilized axons.

$\mu \mathrm{M}$. The number and velocity increased with increasing ATP concentrations and appeared to be maximal at 1 to 5 mM.

In addition to long saltations, a second type of organelle movement was usually seen after the addition of ATP. Particles and mitochondria showed repeated short, jerky, back-and-forth movements oriented parallel to the axon. In most cases, the movements were asymmetrical; movement in one direction (usually the retrograde direc- tion) was faster than in the other direction. Typically, this pattern was repeated several times. The proportion of particles displaying this type of movement usually increased with time while the frequency of long saltations decreased. We believe that these repeated "tugging" movements represent attempts at saltatory movement by a trapped particle. The tugging movements required the presence of ATP and ceased when the ATP was removed. The movements reappeared when ATP was 
TABLE I

Quantitative properties of microscopically visible organelle movements in living lobster motor axons

\begin{tabular}{|c|c|c|}
\hline & Interior $^{a}$ & Periphery $^{a}$ \\
\hline & Mean $\pm S E M$ & Mean $\pm S E M$ \\
\hline \multicolumn{3}{|l|}{$\begin{array}{r}\text { Particle velocity } \\
(\mu \mathrm{m} / \mathrm{sec})^{b}\end{array}$} \\
\hline Retrograde & $1.64 \pm 0.05$ & $1.69 \pm 0.15$ \\
\hline Anterograde & $0.67 \pm 0.04$ & $0.59 \pm 0.02$ \\
\hline \multicolumn{3}{|c|}{$\begin{array}{l}\text { Percentage of organelles moving } \\
\text { in the retrograde direc- } \\
\text { tion }^{c, d}\end{array}$} \\
\hline Particles & $81 \pm 2$ & $31 \pm 3$ \\
\hline Mitochondria & $69 \pm 4$ & $29 \pm 3$ \\
\hline \multirow{2}{*}{\multicolumn{3}{|c|}{$\begin{array}{l}\text { Frequency of movement }{ }^{c} \\
\quad \text { (organelles/min } / 10 \mu \mathrm{m} \text { ) } \\
\text { Particles }\end{array}$}} \\
\hline & & \\
\hline Retrograde & $2.3 \pm 0.3$ & $0.7 \pm 0.07$ \\
\hline Anterograde & $0.5 \pm 0.04$ & $1.80 \pm 0.16$ \\
\hline \multicolumn{3}{|l|}{ Mitochondria } \\
\hline Retrograde & $0.8 \pm 0.1$ & $0.3 \pm 0.04$ \\
\hline Anterograde & $0.3 \pm 0.04$ & $0.6 \pm 0.07$ \\
\hline
\end{tabular}

${ }^{a}$ The microscope was focused approximately in the center of the axon ("Interior") or immediately inside the plasma membrane ("Periphery").

${ }^{b}$ Velocities of saltations were measured from videotape records using a Colorado Video \#610E Video Pointer. The numbers of measurements were: 95 retrograde particles in the interior of 11 axons; 32 retrograde particles at the periphery in 9 axons; 41 anterograde particles in the interior of 11 axons; 44 anterograde particles at the periphery of 7 axons.

'All organelles crossing a vertical line on the video monitor during a 5-min interval were counted from the videotaped record. Elongated organelles more than $1 \mu \mathrm{m}$ long were counted as mitochondria. In most experiments the vertical line on the screen intersected the equivalent of $25.7 \mu \mathrm{m}$ of axon. In order to normalize for different magnifications used in a few experiments, the organelle frequencies are expressed as number of organelles $/ \mathrm{min} / 10 \mu \mathrm{m}$ of vertical line. Results for the interior of the axon are the average of 26 counts in 13 axons. Results for the region immediately below the surface are the average of 14 counts in 11 axons.

${ }^{d}$ By averaging the percentage of organelles moving in the retrograde direction from separate counts, an estimate of the variability between counts can be calculated. However, the estimate of the mean is sensitive to samples in which only a small number of organelles were counted. Another statistic that gives greater weight to samples where larger numbers of organelles were counted is obtained by combining all counts of the organelles moving in each direction. Calculated this way, the percentages of organelles moving in the retrograde direction are: particles in the interior $83 \%$; at the periphery $29 \%$; mitochondria in the interior $71 \%$; and at the periphery $32 \%$.

added again. Similar "tugging" movements of particles and mitochondria were often seen in living axons.

The permeabilization of the plasma membrane by saponin appeared to be irreversible. Intracellular recordings demonstrated that the saponin treatment irreversibly depolarized the membrane. When the internal medium bathing a saponin-treated axon was replaced with external medium the axoplasm rapidly liquefied. The organelles became completely disorganized and displayed Brownian movement in a freely flowing liquid environment. The structural breakdown is probably due more to the chaotropic effects of $\mathrm{Cl}^{-}$ions on the axonal cytoskeleton (Baumgold et al., 1981) than to the action of $\mathrm{Ca}^{2+}$ in depolymerizing microtubules and activating pro-

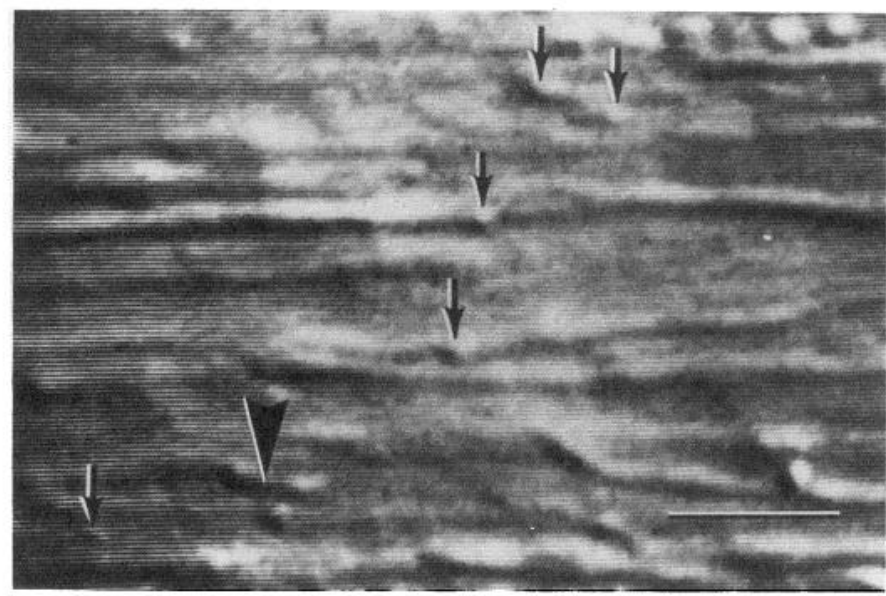

Figure 3. Organelles at the periphery of an axon. The microscope was focused immediately inside the axolemma. Five particles (small arrows) and one small mitochondrion (large ar. rowhead) were moving in the anterograde direction. Bar $=5$ $\mu \mathrm{m}$.

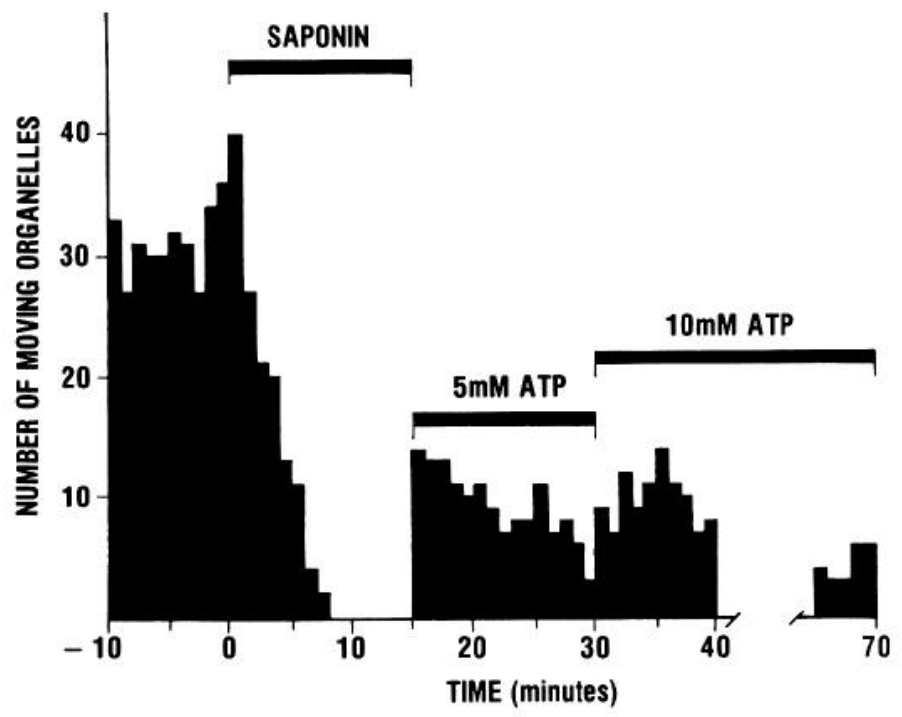

Figure 4. Reactivation of saltatory movement with ATP in a permeabilized axon. The number of organelles displaying movement (either long saltations or "tugging" movements) during successive 1-min intervals was counted from the videotaped record as described under "Materials and Methods." During the $10 \mathrm{~min}$ before the addition of saponin, the axon was pre-incubated in internal medium alone. The time when internal medium containing saponin was added was taken as 0 . After 15 min of saponin treatment, movement was reactivated with saponin-free medium containing $5 \mathrm{~mm}$ MgATP. This was replaced $15 \mathrm{~min}$ later by $10 \mathrm{mM}$ ATP in order to examine the effect of the higher concentration. Movement continued during the period from 40 to $65 \mathrm{~min}$ (data not shown).

teases (Pant and Gainer, 1980). Identical liquefaction was seen in $\mathrm{Ca}^{2+}$-free external medium containing $5 \mathrm{~mm}$ EGTA. On the other hand, if the EGTA in internal medium was replaced by $5 \mathrm{~mm} \mathrm{CaCl} \mathrm{Cl}_{2}$ the axoplasm did not liquefy, but an apparent loosening of structure developed more slowly over a period of 2 to $5 \mathrm{~min}$. Organelles began to show Brownian movement in the radial plane while maintaining their longitudinal position in the 
axoplasm. Mitochondria remained oriented parallel to the long axis. The impression derived from this pattern was that the organelles remained attached to flexible, longitudinally oriented submicroscopic structures but that elements usually cross-linking these structures into a gel had been lost. In spite of this partially disrupted structure, saltatory movements could be reactivated with ATP in the $\mathrm{Ca}^{2+}$-treated axons.

Effect of vanadate on fast transport. Vanadate has been used in studies of cell motility because low concentrations of vanadate ions (in the $\mathrm{V}^{+}$oxidation state) inhibit dynein but not myosin (see "Discussion"). However, vanadate does not readily cross the membranes of living cells (Cande and Wolniak, 1978; Beckerle and Porter, 1982; Forman, 1982a). We confirmed that an hour of incubation in internal or external medium containing $1 \mathrm{~mm}$ vanadate did not noticeably affect organelle transport in intact axons. Nevertheless, vanadate did inhibit ATP-reactivated saltatory movement in permeabilized axons. After the application of $100 \mu \mathrm{M}$ sodium orthovanadate, the number of saltatory movements reactivated by $1 \mathrm{~mm}$ ATP markedly decreased (Fig. $5 A$ ). The long saltations were almost abolished, although some movements (mostly low amplitude tugging movements) remained even with vanadate concentrations as high as 1 mM. The inhibition was rapidly reversible; movement reappeared when the axon was perfused with vanadatefree medium containing $2.5 \mathrm{~mm}$ norepinephrine (Fig. $5 A$ ). The norepinephrine was used because it efficiently reverses vanadate inhibition of dynein by reducing the vanadate (Cande and Wolniak, 1978; Gibbons et al., 1978). Norepinephrine alone $(2.5 \mathrm{~mm})$ did not stimulate ATP-reactivated movement in permeabilized axons that had not been treated with vanadate. The vanadate inhibition could also be reversed by washing with vanadatefree medium without norepinephrine. Substantial inhibition was reproducibly seen with concentrations of vanadate as low as $50 \mu \mathrm{M}$ (Fig. $5 B$ ). Inhibition was not reliably demonstrable in $25 \mu \mathrm{M}$ vanadate (Fig. $5 \mathrm{C}$ ).

Vanadate has been shown to inhibit the $\mathrm{Na}^{+}, \mathrm{K}^{+}$dependent ATPase (Cantley et al., 1977) that forms the sodium pump of the plasma membrane. To determine whether the inhibition of this enzyme by vanadate plays any role in the inhibition of saltatory movement in permeabilized axons, we examined the effect of ouabain, another inhibitor of the $\mathrm{Na}^{+}, \mathrm{K}^{+}$-ATPase. High concentrations of ouabain $(0.25 \mathrm{~mm})$ had no effect on the reaclivation of organelle transport in permeabilized axons.

\section{Discussion}

Microscopic observation of organelle transport in lobster giant axons. The lobster giant motor axon is particularly favorable for optical studies of axonal transport. The axons are relatively easy to isolate and display vigorous saltatory movements that continue for several hours in vitro. The glial sheath interferes less with microscopic visualization than does the myelin of vertebrate axons. Lobster axons have frequently been used for physiological and neurochemical studies (e.g., Kravitz et al., 1965; Freeman et al., 1966), but until recently the advantages of these axons for studies of axonal transport have only rarely been exploited (Smith, 1977; Adams, 1982).
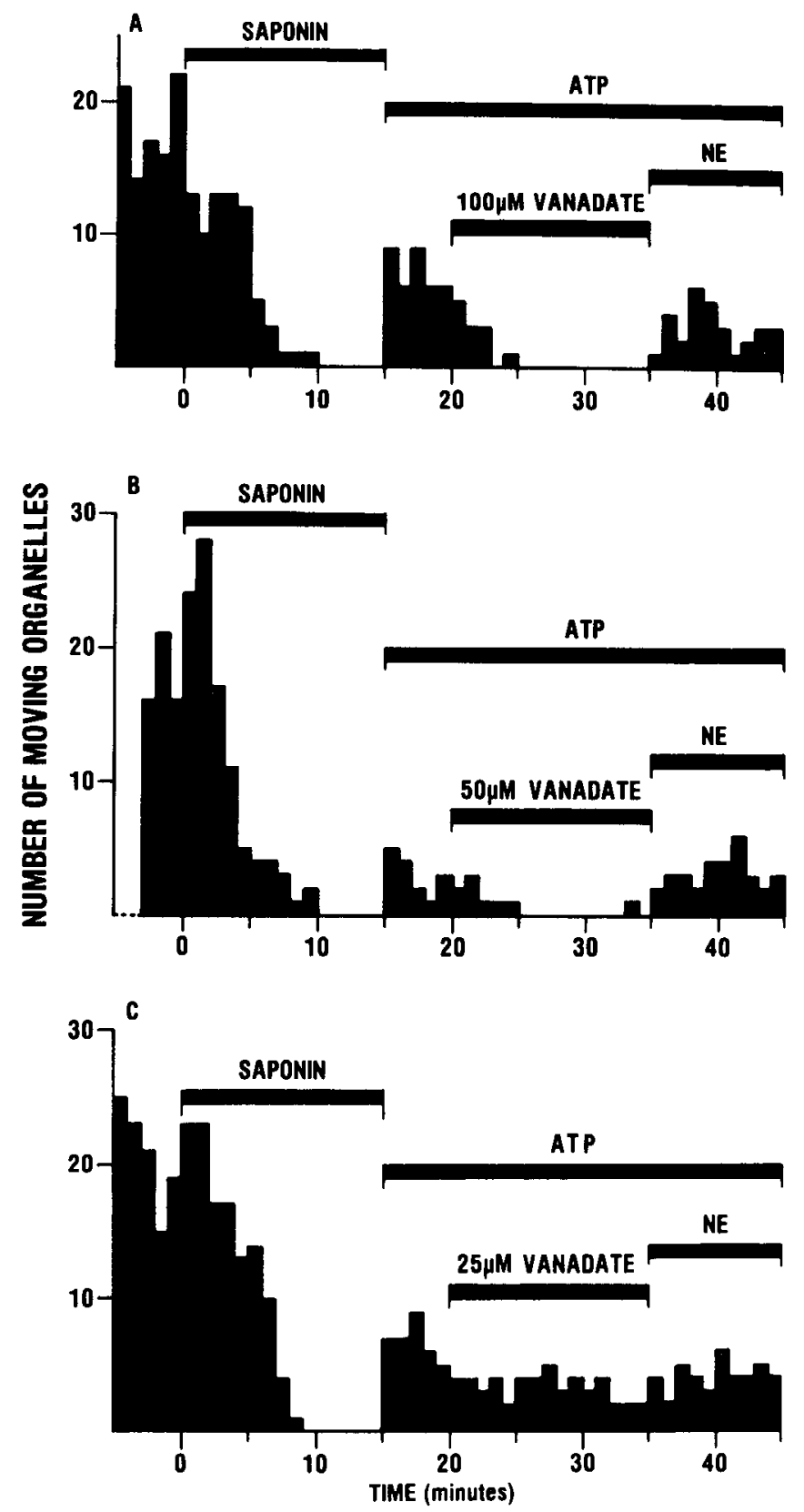

Figure 5. Inhibition of reactivated saltatory movement by vanadate and reversal of the inhibition with norepinephrine $(N E)$. Typical experiments demonstrate the effect of: $A, 100$ $\mu \mathrm{M} ; B, 50 \mu \mathrm{M}$; and $C, 25 \mu \mathrm{M}$ sodium orthovanadate. The frequency of moving organelles was counted as in Figure 4 . When all movement had stopped after $15 \mathrm{~min}$ of saponin treatment, movement was reactivated by the addition of $1 \mathrm{mM} \mathrm{MgATP}$. After $5 \mathrm{~min}$, vanadate was added. Fifteen minutes later the vanadate was removed and $2.5 \mathrm{~mm}$ norepinephrine was added to reduce any residual vanadate. Movement was inhibited by $100 \mu \mathrm{M}$ and $50 \mu \mathrm{M}$ vanadate, but $25 \mu \mathrm{M}$ had little effect.

The organelle movements in lobster axons resemble those previously seen in vertebrate (Smith, 1971, 1972, 1980; Cooper and Smith, 1974; Forman et al., 1977a, b) and crustacean axons (Smith, 1977; Adams, 1982) with conventional light microscopy. It has long been recognized that with conventional light microscopy, only the larger and more refractile organelles were visualized (For- 
man, 1982c). In vertebrate axons, almost all of the particles that had been observed were multivesicular bodies, dense lamellar bodies, and other prelysosomal structures (Smith, 1980; Tsukita and Ishikawa, 1980) that move mainly in the retrograde direction (Smith, 1971, 1972, 1977, 1980; Cooper and Smith, 1974; Forman et al., 1977a). The smaller vesicular and tubulovesicular organelles that are major components of fast anterograde transport (Grafstein and Forman, 1980; Smith, 1980; Tsukita and Ishikawa, 1980) had not been visualized. Recently, however, the improved microscopic contrast and resolution provided by the AVEC-DIC technique (Allen et al., 1981) has made it possible to detect a population of small vesicles that are rapidly transported in the anterograde direction (Allen et al., 1982a, b; Brady et al., 1982; Breuer et al., 1982). Allen and colleagues report that these numerous "small" particles, which apparently correspond to 30- to 50-nm vesicles and tubulovesicular structures (Allen et al., 1982b; Fahim et al., 1982) can be clearly distinguished from a population of "medium" size organelles that are larger and more refractile. Although small particles are reportedly more numerous in lobster axons than medium size particles (Allen et al., 1982a), we were not able to detect structures corresponding to the small particles with our microscopy system, even with an oil immersion objective (numerical aperture $=1.3$ ). Thus, as in all previous studies that did not use the AVEC-DIC technique, the particles described in this paper are all of the "medium" type. The actual size of these "medium" particles cannot be determined by measurement of the video images. Most of the particles are probably close to or below the limit of resolution of the light microscope. Objects more than an order of magnitude smaller than the limit of resolution of the light microscope (a measure of the ability to distinguish two objects as separate) can still be detected. However, the apparent size of objects at or below the resolution limit is inflated by diffraction (Francon, 1961, pp. 35-58; Allen et al., 1981; Forman, 1982c). Preliminary electron micrographs of lobster axons reveal clear vesicles with diameters ranging from 0.1 to $0.5 \mu \mathrm{m}$ (K. Lynch, unpublished data) that apparently correspond to the particles that we observed by light microscopy.

Evidence from radiolabeling studies shows that there are at least two major subcomponents of fast anterograde transport (Lorentz and Willard, 1978; Levine and Willard, 1980; see Grafstein and Forman, 1980). The fastest (transport group I) has been most frequently studied and probably carries synaptic vesicle and plasma membrane precursors (Grafstein and Forman, 1980). The slower component (transport group II) includes mitochondria (Lorentz and Willard, 1978) and nonmitochondrial materials (Levine and Willard, 1980). The smallest particles in lobster axons, those that are detectable only with AVEC-DIC microscopy, have been reported to move in the anterograde direction with a mean velocity of 3.8 $\mu \mathrm{m} / \mathrm{sec}$ (Allen et al., 1982a). These would be expected to form transport group I. The "medium" particles that we observed moving in the anterograde direction had a mean velocity of 0.6 to $0.7 \mu \mathrm{m} / \mathrm{sec}$, which is about the same as that of the anterogradely moving mitochondria. Thereforc, these particles, along with the mitochondria, must move in transport group II. Although transport group I has been extensively studied, very little is known about transport group II. Microscopic study of the medium size, anterogradely moving particles in isolated axons can be used to selectively examine the characteristics of transport group II.

We have found that in lobster axons, anterogradely moving particles and mitochondria are both especially concentrated near the axonal plasma membrane. Predominantly anterograde movement near the surface has also been seen with darkfield optics in other types of crustacean axons (R.S. Smith, personal communication) and with AVEC-DIC microscopy in squid giant axon (Allen et al., 1982b). If anterogradely moving particles are also numerous near the axolemma of vertebrate axons, they may not have been detected due to optical interference from the myelin. In fact, there is autoradiographic evidence that anterograde transport is concentrated close to the plasma membrane of some vertebrate axons (Lentz, 1972; Byers, 1974). Regional differences in the direction of movement in axons might provide clues as to the factors that determine the direction of fast transport.

Organelle transport in permeabilized axons. With the permeabilization technique presented here, giant axons can be used to analyze the biochemical requirements and properties of the transport mechanism. Several of our results demonstrate that the saponin-treated axons have become permeable to small molecules: (1) ATP does not cross the plasma membranes of living cells. In our experiments the very rapid reactivation of movement when ATP is added, the rapid cessation of movement when the ATP is withdrawn, and the rapid reappearance of movement when ATP is again added, all suggest that the membrane must be freely permeable to ATP. (2) Vanadate ions do not readily enter living cells, and incubating intact axons for $1 \mathrm{hr}$ in $1 \mathrm{~mm}$ vanadate had no effect on transport. The inhibition of movement by 50 to $100 \mu \mathrm{M}$ vanadate and the rapid reversal of the inhibition when the vanadate was washed out indicate that the saponintreated axon had become permeable to vanadate. (3) Exposure of the permeabilized axons to external medium with or without $\mathrm{Ca}^{2+}$, or to internal medium containing $5 \mathrm{mM} \mathrm{Ca}{ }^{2+}$, produced rapid changes in axoplasmic structure. Nonpermeabilized axons, on the other hand, function in these same media for many hours without morphological changes. Whether there is an effective upper limit on the size of molecules that can freely enter the saponin-treated axons remains to be determined.

Adams (1982) has recently described a method for reactivating particle transport in giant axons from $\mathrm{Car}$ cinus maenas permeabilized with high voltage discharges. Although the results reported by Adams are generally similar to our findings in lobster axons, there are differences in details between the two studies: (1) Apparently Adams did not detect movement with ATP concentrations below $500 \mu \mathrm{M}$, whereas we detected slight movement at $10 \mu \mathrm{M}$. It may be that we were able to detect more moving particles due to the sensitivity provided by Nomarski optics and video time-lapse recording. (2) Adams reported obtaining inhibition of movement with vanadate concentrations as low as $5 \mu \mathrm{M}$, whereas we 
could not reliably distinguish inhibition below $50 \mu \mathrm{M}$. Adams' experiments with vanadate are not described in detail, and we are unable to explain this discrepancy. (3) Adams reported that 0.5 to $1 \mathrm{~mm} \mathrm{Ca}{ }^{2+}$ causes rapid, protease-dependent dissolution of the axoplasm; we observed a slower, partial disruption of axoplasmic structure. On the whole, however, the degree of agreement between the results of these two independent studies is remarkable.

Inhibition of fast organelle transport by vanadate. Vanadate anions in the $\mathrm{V}^{+}$oxidation state inhibit a variety of ATPases and other enzymes (Cantley et al., 1977; Gibbons et al., 1978; Goodno, 1979). Vanadate has been used as a probe in studies of cell motility because dynein and myosin, the major known mechanochemical coupling ATPases, differ markedly in their sensitivity to vanadate. Low concentrations of vanadate $(0.1$ to $10 \mu \mathrm{M})$ rapidly inhibit isolated flagellar dynein and the dyneindependent beating of cilia and flagella (Cande and Wolniak, 1978; Gibbons et al., 1978; Kobayashi et al., 1978; Hisanaga and Sakai, 1980; Warner and Mitchell, 1980; Shimizu, 1981). This inhibition can be rapidly reversed by removal of the vanadate or by its reduction with reagents such as norepinephrine (Gibbons et al., 1978; Kobayashi et al., 1978; Warner and Mitchell, 1980). On the other hand, vanadate does not significantly inhibit purified myosin except at higher concentrations (500 to $1000 \mu \mathrm{M}$ ) (Cande and Wolniak, 1978; Gibbons et al., 1978; Kobayashi et al., 1978; Goodno, 1979). The inhibition of myosin by vanadate requires several hours to develop and is then irreversible (Goodno, 1979). Furthermore, vanadate does not inhibit well established myosin-based motility such as myofibril contraction (Cande and Wolniak, 1978) or cytokinesis (Cande et al., 1981) in permeabilized cells. Therefore, rapid, reversible inhibition of a motile system by low concentrations of vanadate is consistent with a dynein-based mechanism, rather than one involving myosin (Cande and Wolniak, 1978; Kobayashi et al., 1978). The few cytoplasmic (Hisanaga and Sakai, 1980; Pratt et al., 1980) or membrane-associated (Dentler et al., 1980) dyncins (or dyncin-like molecules) that have been studied have been found to be less sensitive to vanadate than dyneins from ciliary and flagellar axonemes. Nevertheless, the nonaxonemal dynein-like ATPases are reversibly inhibited by vanadate in the range of 10 to $100 \mu \mathrm{M}$, concentrations lower than those needed to inhibit myosin. The sensitivity of dyneins to inhibition by vanadate decreases in conditions of high ionic strength (Hisanaga and Sakai, 1980). The high ionic strength of the medium used for permeabilization may have contributed to our inability to detect inhibition at vanadate concentrations lower than $50 \mu \mathrm{M}$. Thus, the finding that 50 to $100 \mu \mathrm{M}$ vanadate rapidly and reversibly inhibits organelle movement in permeabilized axons suggests that fast axonal transport may involve a dyneinlike molecule. The nucleotide specificity of the reactivated movements (Forman et al., 1982b) is also consistent with a dynein-based mechanism. Because dyneins interact with microtubules, a dynein-based mechanism would be consistent with the considerable body of evidence that microtubules are essential for fast axonal transport (reviewed in Grafstein and Forman, 1980). Nevertheless, it is still possible that an unidentified, vanadate-sensitive protein, different from dynein, is involved in fast axonal transport.

Vanadate also inhibits saltatory organelle movement in several types of permeabilized non-neural cells (Forman, 1981., 1982a, b; Stearns and Boggs, 1981; Beckerle and Porter, 1982; Clark and Rosenbaum, 1982; Stearns and Ochs, 1982). There have been two reports that vanadate fails to block the fast anterograde transport of radioactive proteins when injected into the cell body (Isenberg et al., 1980) or axon (Goldberg, 1982) of invertebrate neurons. However, it is possible that the intracellular vanadate concentration achieved in those experiments was not high enough to inhibit cytoplasmic dyneins (see Forman, 1982a). Furthermore, some cells can overcome the inhibitory effect of microinjected vanadate within 10 to 20 min after injection, probably by reducing the vanadate (Stommel et al., 1980). A brief inhibition by vanadate would not have been detected with the labeling methods. Combining microinjection into living isolated giant axons with direct microscopic observation of the effects might help to resolve some of the discrepancies between the results of microinjection and permeabilization experiments.

Light microscope observation of intra-axonal organelle movement provides information about fast axonal transport with much greater spatial and temporal resolution than can be obtained with other techniques. However, microscopic analysis of transport in vertebrate axons has been hindered by the difficulty of isolating single axons, the delicacy of the axons, and optical interference from the myelin sheath. Large motor axons from lobster walking legs are relatively easy to isolate and survive well in vitro. Saltatory movements of particles and mitochondria are easily visualized in these axons. The permeabilization protocol presented here makes it possible to study the mechanism of transport with probes that cannot enter living axons. The regional difference in the direction of organelle movement may be useful for studying the factors that determine the direction of movement. Thus, isolated giant axons appear to be highly favorable for microscopic studies of the basic mechanisms underlying fast axonal transport.

\section{References}

Abercrombie, R. F., L. M. Masukawa, R. A. Sjodin, and D. R. Livengood (1981) Uptake and release of ${ }^{45} \mathrm{Ca}$ by Myxicola axoplasm. J. Gen. Physiol. 78: 413-429.

Adams, R. J. (1982) Organelle movement in axons depends on ATP. Nature 297: 327-329.

Allen, R. D., N. S. Allen, and J. L. Travis (1981) Video-enhanced contrast, differential interference contrast (AVEC-DIC) microscopy: A new method capable of analyzing microtubulerelated motility in the reticulopodial network of Allogromia laticollaris. Cell Motil. 1: 291-302.

Allen, R. D., R. J. I asek, S. P. Gilbert, A. J. Hodge, and C. K. Govind (1982a) Fast axonal transport in lobster axons. Biol. Bull. 163: 379-380.

Allen, R. D., J. Metuzals, I. Tasaki, S. T. Brady, and S. P. Gilbert (1982b) Fast axonal transport in squid giant axon. Science 218: 1127-1129.

Baumgold, J., S. Terakawa, K. Iwasa, and H. Gainer (1981) Membrane-associated cytoskeletal proteins in squid giant axons. J. Neurochem. 36: 759-764. 
Beckerle, M. C., and K. R. Porter (1982) Inhibitors of dynein block intracellular transport in erythrophores. Nature 295: 701-703.

Brady, S. T., R. J. Lasek, and R. D. Allen (1982) Fast axonal transport in extruded axoplasm from squid giant axon. Science 218: 1129-1131.

Breuer, A. C., P. A. M. Eagles, S. P. Gilbert, R. D. Allen, J. Metuzuals, D. F. Clapin, and R. D. Sloboda (1982) Fast axonal transport in isolated axoplasm of Myxicola infundibulum. Biol. Bull, 163: 381.

Byers, M. R. (1974) Structural correlates of rapid axonal transport: Evidence that microtubules may not be directly involved. Brain Res. 75: 97-113.

Cande, W. Z., and S. M. Wolniak (1978) Chromosome movement in lysed mitotic cells is inhibited by vanadate. J. Cell Biol. 79: 573-580.

Cande, W. Z., K. McDonald, and R. L. Meeusen (1981) A permeabilized cell model for studying cell division: A comparison of anaphase chromosome movement and cleavage furrow constriction in lysed $\mathrm{PtK}_{1}$ cells. J. Cell Biol. 88: 618629.

Cantley, L. C., Jr., L. Josephson, R. Warner, M. Yanigisawa, C. Lechene, and G. Guidotti (1977) Vanadate is a potent $(\mathrm{Na}, \mathrm{K})$ ATPase inhibitor found in ATP derived from muscle. J. Biol. Chem. 252: 7421-7423.

Clark, T. G., and J. L. Rosenbaum (1982) Pigment particle translocation in detergent permeabilized melanophores of Fundulus heteroclitus. Proc. Natl. Acad. Sci. U. S. A. 79: 4655-4659.

Cooper, P. D., and R. S. Smith (1974) The movement of optically detectable organelles in myelinated axons of Xenopus laevis. J. Physiol. (Lond.) 242: 77-79.

Dentler, W. L., M. M. Pratt and R. E. Stephens (1980) Microtubule-membrane interactions. II. Photochemical cross-linking of bridge structures and the identification of a membraneassociated dynein-like ATPase. J. Cell Biol. 84: 311-402.

Fahim, M. A., S. T. Brady, and R. J. Lasek (1982) Axonal transport of membranous organelles in squid giant axons and axoplasm. J. Cell Biol. 95: 330a.

Forman, D. S. (1981) A permeabilized cell model of saltatory organelle movement. J. Cell Biol. 91(Part 2): 414a.

Forman, D. S. (1982a) Vanadate inhibits saltatory organelle movement in a permeabilized cell model. Exp. Cell Res. 141: 139-147.

Forman, D. S. (1982b) Saltatory organelle movement and the mechanism of fast axonal transport. In Axoplasmic Transport (Proceedings in the Life Sciences, Vol. 1), D. G. Weiss, ed., pp. 234-240, Springer-Verlag, Heidelberg.

Forman, D. S. (1982c) Microscopic methods for the observation of axonal transport in living axons. In Axoplasmic Transport (Proceedings in the Life Sciences, Vol. 1), D. G. Weiss, ed., pp. 424-428, Springer-Verlag, Heidelberg.

Forman, D. S., and W. G. Shain, Jr. (1981) Batrachotoxin blocks saltatory organelle movement in electrically excitable neuroblastoma cells. Brain Res. 211: 242-247.

Forman, D. S., A. L. Padjen, and G. R. Siggins (1977a) Axonal transport of organelles visualized by light microscopy: Cinemicrographic and computer analysis. Brain Res. 136: 197-213.

Forman, D. S., A. L. Padjen, and G. R. Siggins (1977b) Effect of temperature on the rapid retrograde transport of microscopically visible intra-axonal organelles. Brain Res. 136: 215-226.

Forman, D. S., K. J. Brown, and D. R. Livengood (1982a) Fast axonal transport in permeabilized lobster giant axons. Soc. Neurosci. Abstr. 8: 827.

Forman, D. S., K. J. Brown, M. R. Adelman, and D. R. Livengood (1982b) Nucleotide specificity of fast axonal transport in permeabilized lobster giant axons. J. Cell Biol. 95 (Part 2):
$323 a$.

Francon, M. (1961) Progress in Microscopy, Pergamon Press, New York.

Freeman, A. R., J. P. Reuben, P. W. Brandt, and H. Grundfest (1966) Osmometrically determined characteristics of the cell membrane of squid and lobster giant axons. J. Gen. Physiol. 50: 423-445.

Gibbons, I. R., M. P. Cosson, J. A. Evans, B. H. Gibbons, B. Houck, K. H. Martinson, W. S. Sale, and W. -J. Y. Tang (1978) Potent inhibition of dynein adenosinetriphosphatase and of the motility of cilia and sperm flagella by vanadate. Proc. Natl. Acad. Sci. U. S. A. 75: 2220-2224.

Goldberg, D. J. (1982) Microinjection into an identified axon to study the mechanism of fast axonal transport. Proc. Natl. Acad. Sci. U. S. A. 79: 4818-4822.

Goodno, C. C. (1979) Inhibition of myosin ATPase by vanadate ion. Proc. Natl. Acad. Sci. U. S. A. 76: 2620-2624.

Grafstein, B., and D. S. Forman (1980) Intracellular transport in neurons. Physiol. Rev. 60: 1167-1283.

Hisanaga, S. -I., and H. Sakai (1980) Cytoplasmic dynein of the sea urchin egg. I. Partial purification and characterization. Dev. Growth Differ. 22: 373-384.

Isenberg, G., P. Schubert and G. W. Kreutzberg (1980) Experimental approach to test the role of actin in axonal transport. Brain Res. 194: 588-593.

Kirkpatrick, J. B., J. J. Bray, and S. M. Palmer (1972) Visualization of axoplasmic flow in vitro by Nomarski microscopy. Comparison to rapid flow of radioactive proteins. Brain Res. 43: $1-10$.

Kobayashi, T., T. Martensen, J. Nath, and M. Flavin (1978) Inhibition of dynein ATPase by vanadate, and its possible use as a probe for the role of dynein in cytoplasmic motility. Biochem. Biophys. Res. Commun. 81: 1313-1318.

Kravitz, E. A., S. W. Kuffler, D. D. Potter, and N. M. van Gelder (1965) Gamma-aminobutyric acid and other blocking compounds in crustacea. II. Peripheral nervous system. J. Neurophysiol. 12: 323-328.

Lentz, T. L. (1972) Distribution of leucine- ${ }^{3} \mathrm{H}$ during axoplasmic transport within regenerating neurons as determined by electronmicroscopic radioautography. J. Cell Biol. 52: 719-732.

Levine, J., and M. Willard (1980) The composition and organization of axonally transported proteins in the retinal ganglion cells of the guinea pig. Brain Res. 194: 137-154.

Lorentz, T., and M. Willard (1978) Subcellular fractionation of intra-axonally transported polypeptides in the rabbit visual system. Proc. Natl. Acad. Sci. U. S. A. 75: 505-509.

Pant, H. C., and H. Gainer (1980) Properties of a calciumactivated protease in squid axoplasm which selectively degrades neurofilament proteins. J. Neurobiol. 11: 1-12.

Pratt, M. M. (1980) The identification of a dynein ATPase in sea urchin eggs. Dev. Biol. 74: 364-378.

Pratt, M. M. T. Otter, and E. D. Salmon (1980) Dynein-like $\mathrm{Mg}^{2+}$-ATPase in mitotic spindles isolated from sea urchin embryos (Strongylocentrotus droebachiensis). J. Cell Biol. 86: 738-745.

Rebhun, L. I. (1972) Polarized intracellular particle transport: Saltatory movements and cytoplasmic streaming. Int. Rev. Cytol. 32: 93-137.

Shimizu, T. (1981) Steady-state kinetic study of vanadate-induced inhibition of ciliary dynein adenosinetriphosphatase activity from Tetrahymena. Biochemistry 20:4347-4354.

Smith, R. S. (1971) Centripetal movement of particles in myelinated axons. Cytobios 3: 259-262.

Smith, R. S. (1972) Detection of organelles in myelinated axons by dark field microscopy. Can. J. Physiol. Pharmacol. 50: 467-469.

Smith, R. S. (1977) Rapid particle transport in decapod crustaceans. Soc. Neurosci. Abstr. 3: 31. 
Smith, R. S. (1980) Short term accumulation of axonally transported organelles in the region of localized lesions of single myelinated axons. J. Neurocytol. 9: 39-65.

Stearns, M. E., and D. P. Roggs (1981) Studies of axonal transport in digitonin permeated neuroblastoma cells. J. Cell Biol. 91: 420a.

Stearns, M. E., and R. L. Ochs (1982) A functional in vitro model for studies of intracellular motility in digitonin permeabilized erythrophores. J. Cell Biol. 94: 727-739.

Stommel, E. W., R. E. Stephens, and D. L. Alkon (1980) Motile statocyst cilia transmit rather than directly transduce mechanical stimuli. J. Cell Biol. 87: 652-662.
Tsukita, S., and H. Ishikawa (1980) The movement of membranous organelles in axons. Electron microscopic identification of anterogradely and retrogradely transported organelles. $J$. Cell Biol. 84: 513-530.

Warner, F. D., and D. R. Mitchell (1980) Dynein: The mechanochemical coupling adenosine triphosphatase of microtubulebased sliding filament mechanisms. Int. Rev. Cytol. 66: 1-43. Wiersma, C. A. G. (1961) The neuromuscular system. In The Physiology of Crustacea, T. H. Waterman, ed., Vol. 2, pp. 191-240, Academic Press, New York.

Willingham, M. C., and I. Pastan (1978) The visualization of fluorescent proteins in living cells by video intensification microscopy (VIM). Cell 13: 501-507. 